J. Lake Sci. (湖泊科学), 2014, 26(6):813-821

http://www. jlakes. org. E-mail : jlakes@niglas.ac.cn

(c) 2014 by Journal of Lake Sciences

\title{
水位变化对河流、湖泊湿地植被的影响”
}

\author{
姚 釒金 $^{1}$,杨桂山 ${ }^{2}$,万荣荣 ${ }^{2}$, 王晓龙 ${ }^{2}$ \\ ( 1 : 南京信息工程大学遥感学院,南京 210044) \\ (2: 中国科学院南京地理与湖泊研究所湖泊与环境国家重点实验室,南京 210008)
}

摘 要: 水位是湿地生态水文过程的关键因素之一, 其改变将影响湿地植被覆盖度和物种组成, 最终产生群落演替. 从水 位梯度,水位波动和人工控湖、控河工程 3 方面论述水位变化对湿地植被的影响: 由于对水位选择的不同及彼此竞争力的 差异, 湿地植物种沿水位具有梯度分布现象, 同时形态可塑性能对其分布范围产生一定影响; 水位波动的频率和淹没持 续时间对于植被演替具有基础性的作用,水位波动幅度的影响则相对较小, 周期性波动能维持以草本植物为主的湿地植 被的物种多样性和稳定性, 非周期性波动以洪水、干旱为主, 易促进湿地植被向固定的水生或陆生方向演替; 人工控湖、 控河的影响在机理上并无特殊之处,但保证物种多样性和生态系统稳定性的各种缓解措施具有较高的参考价值. 基于机 理的量化模型,自然、人为因素驱动下水位变化对湿地植被影响的差别研究, 模拟水位波动实验以及人工控湖、控河工程 的跟踪观测将是今后该领域研究的热点.

关键词: 水位;湿地植被;物种多样性;人工控湖、控河;水位调控

\section{Impact of water level change on wetland vegetation of rivers and lakes}

\section{YAO Xin ${ }^{1}$, YANG Guishan ${ }^{2}$, WAN Rongrong ${ }^{2} \&$ WANG Xiaolong ${ }^{2}$}

( 1 : Department of Remote Sensing, Nanjing University of Information Science and Technology, Nanjing 210044, P. R. China)

(2: State Key Laboratory of Lake Science and Environment, Nanjing Institute of Geography and Limnology, Chinese Academy of Sciences, Nanjing 210008, P. R. China)

Abstract: Water level is one of the key factors of the eco-hydrological process in wetlands. The change of water level often results in variations in wetland vegetation coverage and species composition, and leads to community succession. This article summarized the impact of water level on wetland vegetation in three different aspects: water level gradient, water level fluctuations and artificial lake/river control projects. The results showed that: 1) Wetland species exhibited grade distribution along water level gradient mainly due to their distinct responses to water level and their different competitiveness. Meanwhile, morphological plasticity influenced the distribution range of individual species; 2 ) The rate and duration of water level fluctuations, rather than depth, had important influence on vegetation succession; periodic water level fluctuations maintained the species diversity and ecological stability, however, a periodic water level fluctuations, mainly the flood and drought, were likely to cause vegetation succession from wetland ecosystems to terrestrial or aquatic ecosystems; 3 ) Artificial lake/river control projects had the same impact with water level fluctuations in theory, however, the matching mitigation strategies provide valuable references for maintaining ecological diversity and stability. The potential aspects to be attached importance in the future of the research are as follows: quantitative models based on mechanisms, differentiating impacts caused by anthropogenic and natural driving factors, simulating the water level fluctuations in laboratories and the tracking attention on the long-term impact of artificial lake/river control projects.

Keywords: Water level; wetland vegetation; species diversity; artificial lake/river control; water level management

湿地的水文过程面临人类活动、气候变化等诸多方面的影响,其所支撑的湿地生态系统也因此发生不 同程度的变化 ${ }^{[1]}$.水位作为湿地水文过程的最主要因素 ${ }^{[2]}$, 对湿地植被覆盖度、物种组成、初级生产力及内

* 国家自然科学基金青年基金项目 (41301035) 和国家重点基础研究发展计划 “973” 项目 (2012CB417006) 联合资 助. 2013-08-21 收稿;2014-05-12 收修改稿. 姚金金(1982 ), 男,博士, 讲师; E-mail: yaoxin@ nuist. edu. cn. 
部物质能量循环起着至关重要的调节作用,其改变能对整个湿地生态系统的结构和功能的稳定性产生深刻 而持久的影响 ${ }^{[3]}$. 同时, 水位变化对湿地植被的影响在构成 “气候变化/人类活动一湿地环境 (土壤、水文)一 湿地生态” 的研究环节中具有基础性,因而成为目前湿地生态水文学及全球变化共同关注的热点 ${ }^{[4-5]}$.

$1980 \mathrm{~s}$ 前, 已有部分学者从植物学的角度考虑水位变化对湿地植物种 (水生、湿生) 的影响, 研究主要考 虑不同水深的溶解氧差别以及因此产生的植物生长状况差异等 ${ }^{[6]} ; 1980 \mathrm{~s}$ 后, 随着生态学的发展和长时间序 列水文数据的积累, 用生物多样性、覆盖度等生态学指标研究自然条件下水位波动 (主要是洪水、干旱) 对湿 地植被的影响成为新的研究热点 ${ }^{[7-8]} ; 1990 \mathrm{~s}$ 以来, 人工控湖、控河的生态效应逐渐为世人所关注, 不少研究 提出了基于植被稳定性的水位调控方案作为水库、水坝等工程建设和运行的决策支持和参考建议 ${ }^{[9-10]}$. 因 此, 迄今为止国内外关于水位变化与湿地植被关系的大量工作, 按照研究目的及内容的差别, 可以归纳为以 下 3 个方面: 水位梯度对湿地植被的影响, 水位波动对湿地植被的影响以及人工控湖、控河工程的生态效应 研究.

\section{1 水位梯度对湿地植被的影响}

\section{1 不同植物种沿水位的梯度分布}

湿地植物种沿水位具有梯度分布现象, 当水位以水深 (植物生长点的水面与地表之间距离) 度量时, 随 着水深的增加, 植物群落优势种将经历 “旱生一湿生一水生” 的转变 ${ }^{[1-12]}$, 同时群落多样性先增后减, 在湿生 植物群落中达到最大值. 对于这一现象, 不同学者从各个角度给予了解释.

从物种生存对策的宏观角度分析, 不同物种的梯度分布是由物种适应环境的能力决定的,这些能力包 括竞争对策、杂草对策、耐逆境对策 $(C 、 R 、 S)$ 等. Rorslett ${ }^{[13]}$ 据此提出了综合的植被策略指数, 并将其运用于 挪威 17 个湖泊的沉水植被交替研究, 确认了植物种沿水位的梯度变化, 同时认为拥有杂草对策的物种对水 位梯度的适应范围更广。

更深人的微观研究发现, 多数植物种在水位选择上具有趋异性 ${ }^{[14-15]}$. Keddy 等 ${ }^{[14]}$ 以 11 种湿地植物沿水 位梯度进行室内培养, 分析其对水位的选择情况, 实验结果与野外调查情况基本一致, 即对水位梯度反应明 显的 6 种植物种适宜生长的水位多数不同 (表 1 ).

表 1 湿地植物种子萌发对水位梯度的响应

Tab. 1 The response of wetland plant species seedling recruitment to water level gradient

\begin{tabular}{|c|c|c|c|}
\hline 湿地植物 & 野外分布特征 & 水位梯度反应 $(\mathrm{T} / \mathrm{F}) *$ & 最适萌发水位 $/ \mathrm{cm}$ \\
\hline 草原网茅(Spartina pectinata) & 水面以上 & $\mathrm{T}$ & -2 \\
\hline 水菖蒲 (Acorus calamus) & 水面以上 & $\mathrm{T}$ & $\leqslant-5$ \\
\hline 斑叶苶( Polygonum punctatum) & 水面以上 & $\mathrm{T}$ & -2 \\
\hline 柳叶鬼针草 (Bidens cernua) & 水面以上 & $\mathrm{T}$ & 0 \\
\hline 泽泻 (Alisma plantago-aquatica) & 浅水区 & $\mathrm{T}$ & $\geqslant 10$ \\
\hline 贯叶泽兰 (Eupatorium perfoliatum) & 水面以上 & $\mathrm{T}$ & 1 \\
\hline 花叶水苾 (Scirpus validus) & 浅水区 & $\mathrm{F}$ & - \\
\hline 制椅草(Scirpus americanus) & 浅水区 & $\mathrm{F}$ & - \\
\hline 宽叶慈姑 (Sagittaria latifolia) & 浅水区 & $\mathrm{F}$ & - \\
\hline 水烛 (Typha angustifolia) & 水面以上 & $\mathrm{F}$ & - \\
\hline 千屈菜( Lythrum salicaria) & 水面以上 & $\mathrm{F}$ & - \\
\hline
\end{tabular}

$* \mathrm{~T}$ 代表对水位梯度反应明显, $\mathrm{F}$ 代表对水位梯度反应不明显.

对水生植物而言, 水深的不同意味着植物可利用的光能和水体溶解氧有较大差异 ${ }^{[16]}$, 从而影响到植物 的光合作用 ${ }^{[17]}$, 不同物种对光和溶解氧的需求不一, 如淡水湖泊常见的菹草、狐尾藻、大茨藻、金鱼藻、苦草 等水生植物种, 其光补偿点差别可达数倍以上 ${ }^{[18]}$, 故最终呈现对水位的不同选择. 对湿生植物如香蒲、芦苇 等而言, 根系组织需要出露的茎叶部分提供足够的氧, 水深增加会导致植物内部输氧能力的变化, 较深的水 中甚至出现由于根系需求过大, 茎叶自身氧浓度偏低的现象, 从而影响其正常生理功能 ${ }^{[19-20]}$, 同时湿生植物 
淹没与否, 光合作用差异是非常显著的 ${ }^{[21]}$. 也有部分学者认为, 水深变化改变了土壤的水分、盐碱度 ${ }^{[22]}$, 进 一步改变了以此为基础的物质循环、能量流动,对湿生植物群落分布也非常重要.

一些物种之间生物学特性类似, 室内培养对水位的选择呈现趋同性, 野外分布的差异可能仅仅是竞争 导致. Keddy 等 ${ }^{[23]}$ 据此提出了物种分布的离心组织模型 (centrifugal organization model), 认为当植物种对于 水位的选择趋势一致时, 最具竞争力的物种处于最佳水位范围, 其他物种按照竞争力大小, 以此水位为中心 呈梯度分布.

综上,湿生植物沿水位梯度分布的现象实际上可以用生态幅相关理论加以解释:生理最适点差异是最 主要原因,生理最适点趋同时则由生态最适点差异导致. 此外就短期而言, 还需适当兼顾种子库的影响 ${ }^{[24]}$.

\section{2 植物种对水位梯度的适应}

野外调查表明,多数植物种在偏离其最适宜生存水位的地区也可萌发、生长 ${ }^{[14,25]}$, 植物这种适应不同水 位的能力主要取决于其形态的可塑性及内部营养物质分配的调整 ${ }^{[26]}$.

Coops 等 ${ }^{[27]}$ 对湿生植物的研究表明, 生长于深水处的芦苇和莎草的地上、地下部分生物量比和根茎直 径显著增加, Vretare 等 ${ }^{[28]}$ 和 Blanch 等 ${ }^{[29]}$ 的结论与此一致, 同时认为处于深水位的植物扎根更靠近土壤表 层, 且拥有更高的相对生长速率值 (relative growth rate, RGR). Lentz 等 ${ }^{\left[{ }^{[30]}\right.}$ 对水生植物的研究发现, 处于深水 处的植株最大叶片高度在生长期高于浅水处植株, 有利于其进行光合作用. 此外, 不同植物实现形态可塑性 的方式不同, 决定了其适应能力的差别,如莎草科的 Baumea arthrophylla 通过改变茎的数量和高度, 缓慢进 行调整;而水冬麦 Triglochin procerum 则能以生成、脱落海绵叶的方式快速调整 ${ }^{[31]}$.

以上这些适应方式与水环境的特点是分不开的, 即弱光、缺氧, 无论是叶片、根系还是通气组织的调整 变化都是为了从这两个方面满足植物生长的需求.

\section{3 水位梯度与植被分布的量化关系}

Weiher 等 ${ }^{[32]}$ 曾对 120 个湿地微生境中 20 种植物进行了为期 5 年的跟踪调查, 发现植物的分布除了跟 水位等生境因子有关外,也存在一定的随机性,这导致预测物种分布较为困难.

然而在一些特定情况下, 基于统计结果的适度量化对于宏观研究仍有很多便利之处, 吴业刚等 ${ }^{[33]}$ 在研 究香蒲对莎草群落的人侵时将湿地以栅格划分, 提出了湿地物种演替的马尔科夫模型, 认为莎草群落转变 为香蒲群落的概率 $P_{\mathrm{e}}$ 为:

$$
P_{\mathrm{e}}=\frac{1}{1+\alpha \cdot \mathrm{e}^{-\beta \cdot P}}+\gamma \frac{D}{P}
$$

式中, $\alpha \beta \beta \gamma$ 为模型系数, $D$ 为水深, $P$ 与水体磷浓度相关. 因为此处只涉及两个物种, 所以精度有一定保证.

另一些研究验证了植物种的分布特征 (如丰富度、盖度、根系密度及生物量等) 与水位梯度的关系用 Gussian model 能很好地模拟,其方程为:

$$
y=c \cdot \exp \left[-\frac{(x-u)^{2}}{2 t^{2}}\right]
$$

式中, $y$ 表示植物种相关的指标,即前述的丰富度、盖度等; $c$ 为物种指标在研究区内的最优化值; $x$ 为水位梯 度; $u$ 为对应于 $c$ 取值的水位梯度值; $t$ 是一个与阈值相关的量 ${ }^{[34-37]}$.

得益于 $3 \mathrm{~S}$ 技术的进步,此类基于景观生态的空间显式 (spatial explicit) 的模型方法仍在不断地成熟发展 中 $^{[38-39]}$, 进一步优化的可能在于考虑更周全的生态因子以及输人更详细的 DEM 数据 ${ }^{[40]}$. 尽管如此, 由于水 位对植物种的影响属于间接作用, 其间涉及多种物质循环、能量流动过程, 水位梯度与植被分布关系量化研 究的进展并不简单,已有研究结论在不同类型湿地生态系统中的适用性也有待进一步验证.

\section{2 水位波动对湿地植被的影响}

\section{1 人工模拟水位波动对湿地植物种的影响}

与水位梯度相比,水位波动是一个复杂的过程,包含参数较多, 导致实验室模拟难度较大, 同时自然条 件下水文事件如干旱、洪水等,在规模、影响尺度上更是模拟实验难以企及的. 尽管如此,在分项研究水位波 
动的不同要素(如水位波动的波幅、时刻、持续时间、频率等) 对湿地植被影响的机理方面, 人工模拟实验仍 有独到之处.

Casanova 等 ${ }^{[41]}$ 的研究具有一定代表性, 他将水位波动以最大深度变幅、淹没持续时间和频率 3 因素表 征, 通过测定种子库的物种丰富度及生物量, 认为淹没持续时间和频率共同决定了湿地植被由陆生种到湿 生、水生种的转变, 而最大深度变幅的作用较小, 其作用主要在于影响植物种是波动耐受型 ( fluctuation-tolerator) 还是波动响应型 (fluctuation-responder). 除此之外, Vretare 等 ${ }^{[28]}$ 设置不同水位变幅表征波动, 认为较大 水位变幅会导致植物相对生长速率减小, Nicol 等 ${ }^{[42]}$ 以水位变化速率单因素模拟退水对湿地种子库的影响, 认为退水速度较快有利于陆生植物种建群. 目前尽管 WLF (water-level fluctuations) 三要素分解已被广泛接 受 ${ }^{[43]}$,但从各要素相互影响及植物种综合反应的角度来看, 此方面研究仍有较大发展空间.

\section{2 自然条件下水位波动对湿地植被的影响}

自然界水位波动情况千差万别, 可以粗略地分为以下两类: 周期性波动, 如季节性涨落水湖泊 (鄱阳湖 等); 非周期性波动,主要体现为洪水和干旱.

2.2. 周期性水位波动对湿地植被的影响 周期性水位波动以高水位和低水位的反复交替出现为特征, 对 整个湿地的植被组成及分布有决定性作用. 以河、湖滨带湿地为例, 高水位的淹没使湿地原有优势物种大量 死亡, 当水位消退后, 多种物种得到了萌发、生长的空间, 对于木本植物而言, 因为其生命周期较长, 在短暂 的退水期间不易重新建群, 容易为短生命史的草本植物取代, 所以水位的周期波动在河、湖滨带形成了大面 积的草本湿地群落, 维持了湿地丰富的物种多样性 ${ }^{[7]}$.

Vangeest 等的结论与此类似, 对荷兰 215 个湖泊的调查表明, 当湖泊年龄增加导致底部水力连通性下 降, 使水位波动减少后, 湖滨带植被类型由耐水位波动型转为对水位波动敏感型, 多样性也会随之减少 ${ }^{[44]}$. 高低水位交替变化有利于维持湿地生物多样性、r 策略物种生存的结论已经基本达成共识.

此外, Riis 等 ${ }^{[45]}$ 对新西兰 21 个湖泊湖滨植被群落的调查发现, 当水位变幅接近 $1 \mathrm{~m}$ 时, $30 \mathrm{~d}$ 左右的出 露期对维持当地植物群落物种多样性最为有利; Blanch 等 ${ }^{[46]}$ 的研究发现, 湿地植物丰富度与水位波动过程 中水深处于 $0 \sim 60 \mathrm{~cm}$ 间的淹没时间及 $1 \mathrm{~m}$ 以上的出露时间相关. 这些研究表明, 水位波动幅度和持续周期 不同时,水位波动的影响效果也存在明显差别.

2.2.2 非周期性水位波动对湿地植被的影响 自然界非周期性的水位波动以长期洪水或长期干旱为主, 其 效果趋向于永久淹没或永久排干, 导致湿地向水生或陆生生态系统转变, 同时伴随植物物种的减少和生物 多样性的下降. 如 Gacia 等 ${ }^{[47]}$ 发现, 在一次持续时间较长的洪水过后, 随着水面上升 $5.5 \mathrm{~m}$, 原有生长于距水 面 $0.6 \mathrm{~m}$ 左右的水非属植物位置上移, 新的生存高度也相应提升 $5.3 \sim 5.5 \mathrm{~m}$ 左右; Vandervalk 等 $^{[48]}$ 对水位 永久上升后湿地植被的调查表明, 样区的物种数、根系密度和 Shannon-Wiener 多样性指数全面下降. 于丹 ${ }^{[49]}$ 对哈尔滨西郊的沼泽湿地 1982-1991 年为期 10 年的研究发现, 水体淤积导致了沼泽旱化过程, 使湿地植被 的演替序列为: 沉水型一浮叶型一高大挺水型一矮小挺水型. Laine 等 ${ }^{[50]}$ 对排干年份为 $3 \sim 55$ 年的不同沼泽湿 地进行研究, 发现了以莎草种消失为显著特征的沼生植被向森林植被演替的过程, 同时伴随着整个研究区 的 $\gamma$ 多样性显著降低,尽管对个别样点而言, 由于微生境的影响, $\alpha$ 多样性的变化可能不太明显.

\section{3 水位波动引起的环境变化对湿地植被的间接影响}

水位波动某种程度上会影响湿地植被的生存环境, 从而对其个体生长、形态及群落组成、动态起到一定 的影响. 有研究表明, 浅水湖泊、河流的水位波动易导致底泥的悬浮, 从而产生轻度的富营养化, 同时水体的 浊度、透光率也会发生改变, 这些都对水生植物物种的丰富度、多样性等指标产生间接影响 ${ }^{[51-53]}$. 对湖滨带 和河岸带的湿生植物而言, 周期的淹水后土壤物化性质会发生改变, 如土壤氧化还原电位下降、土壤氧含量 下降和根系压力的增加, 进一步导致植物的光合作用相关酶的活性降低 ${ }^{[54]}$. 此外, 水位波动会导致土壤有机 质分解速率下降, 使土壤中可用营养物质减少, 也会影响湿地植物对营养物质的吸收, 使其生长变缓 ${ }^{[55]}$, 同 时也会影响土壤营养元素与水体间的交换过程,该过程因高程差异而不同 ${ }^{[56]}$.

\section{4 其他环境因素对水位波动影响的干扰}

水位波动对湿地植被作用的程度容易受其他环境因素的影响. 如 Keeland 等 ${ }^{[57]}$ 认为初始水位对水位波 动的影响至关重要, 处于土壤水分饱和区和常年淹没区的一些较高的湿地植物的生长与水位波动无显著关 
系, 而较干旱地区的同种植物对水位波动则有明显反应, 原因可能是初始水位改变了物种对水位的适应能 力, 使其在水位波动时表现各异. Lenssen 等 ${ }^{[58]}$ 以 6 种湿地植物对土壤营养盐和水位波动的交互作用做了详 细的研究, 发现在较贫㾑的矿化基质中, 水位波动只影响 2 种植物的生长, 而在腐殖质中, 水位波动的影响 覆盖全部 6 种植物, 这表明当营养盐浓度过低, 成为湿地植物生长的限制因子时, 水位波动对植被的影响将 退居其次.

综上所述, 水位波动对湿地植被既有直接影响也有间接影响 (严格意义上, 水位变化对光照、溶解氧的 改变也是间接影响), 既可以是限制因子也可以是非限制因子, 此外波动本身难以定量, 更增加了其研究难 度. 人工模拟和自然条件下的研究实际上是分别从植物生理和生态两个角度探讨水位波动的影响, 前者易 于解释微观机理, 后者更擅长宏观上把握植被变化过程, 各有所长也各有局限, 相互结合才能优势互补.

\section{3 人工控湖、控河工程的生态效应}

人工控湖、控河工程改变了湖泊、河流湿地原有的水文状况, 导致水位波动的次数和规模发生变化,使 湿地植被朝着新的方向演替,在一定时间后形成另一种类型的稳定的群落形态和结构. 与自然水位波动的 影响研究相比,人工控湖、控河工程在机理上并无特殊之处,但国外在控湖、控河实施方案的论证方面往往 比较严格, 无论是影响的分析调查, 还是缓解对策的提出, 都较为完整、系统, 此外中欧在控湖方面有长达数 百年的历史, 有许多经验值得借鉴 ${ }^{[59]}$.

\section{1 人工控湖、控河工程对湿地植被的影响}

出于不同目的进行人工控湖、控河时, 其水文机制的改变方式也不同, 这些改变包括永久性抬升或降低 水位 ${ }^{[10,44,60-62]}$ 、增强或削弱水位变幅 ${ }^{[63-66]}$ 、增加或减少水位波动频率 ${ }^{[67-68]}$ 等, 不同的改变方式对湿地植被的 影响也不同. 多种调控方式及其综合使水位波动相关变量极其复杂, 更为重要的是,湿地植被的形成是多次 调控的结果. 尽管如此, Toner 等 ${ }^{[63]}$ 认为, 少数变量对人工调控下湿地植被类型的演替起着至关重要的作用, 如最近一次水位波动的起、止时刻及上一次水位波动的结束时刻, 并据此建立了精确度较高的湿地植被从 草本到木本的演替预测模型.

分别针对人工调控的水位、波动幅度及频率进行进一步研究, 这正是自然水位波动对湿地植被的影响 研究难以实现的. 无论初始水位如何, 水位的永久性抬升或降低,会导致湿地物种多样性的减少和植被覆盖 度降低,随着时间的推移, 部分地区的湿地植被会慢慢恢复以适应新的水位 ${ }^{[69]}$,但也有地区湿地植被长时间 后仍无法自行恢复 ${ }^{[60]}$; 水位波动幅度的增加或减少对湿地植被的影响不尽相同, 从无波动到高波动, 植被多 样性经历先增加后减少的过程, 对于多数调控而言, 约 1 2 m 的年内变幅对湿地多样性的增加和稳定最为 有利 ${ }^{[65,70]}$, 这与前文提及的 Riis 和 Blanch 等的研究结论有一定的相似处 ${ }^{[4546]}$; 快速、变幅较小的日波动和 慢速但变幅较大的年内波动都会造成群落多样性下降,但造成多样性下降的更重要的原因是水库、水坝的 蓄水状况对水位的改变 ${ }^{[67]}$.

水位调控下植被群落特征的整体变化主要源自植物种对水位波动的反应, 植物的种子散布机制对于其 是否适应水位调控相当重要 ${ }^{[71]}$, 具有风媒、水媒散布机制的物种更容易在频繁的水位调控过程中存活, 同时 本地种的减少为外来种提供了生存空间, 使植被群落易受外来种人侵, 但整体而言, 频繁的水位调控会使物 种数和植被覆盖处于下降趋势 ${ }^{[67]}$.

\section{2 缓解对策}

为了缓解水位调控带来的负面影响, 不少地区在对影响进行调查、论证后, 从保护湿地植物多样性、稳 定湿地生态系统的角度出发,对现行水位调控方案进行调整,或提出了相应缓解措施. 制定一套完善的水位 调控方案和缓解对策不仅需要对湿地生态系统演替与水位变化之间的关系有完整的把握, 同时要了解基于 生态、经济等不同基础的河流、湖泊需水量, 以及各地区之间的差异,包括土壤状况、海拔和经纬度决定的植 物生长期长短、管理前的水位波动状况、植被状况等 ${ }^{[72]}$. 已有研究表明,即使对邻近的湿地实施同样的调控 方案, 其效果也可能截然相反, 所以因地制宜地制定调控方案和缓解措施相当重要 ${ }^{[61]}$. 另外, 缓解措施能否 达到预期的效果也需要实地跟踪调查并及时修改调整. 表 2 列出了国外不同地区控湖、控河的生态影响及 采取的一些缓解措施, 以供参考 ${ }^{[10,61-62,66-68,73]}$. 
表 2 国外不同地区控湖、控河工程的生态影响及缓解措施

Tab. 2 Ecological impacts and mitigation strategies of artificial lake/river control projects

in different districts in foreign countries

\begin{tabular}{|c|c|c|c|c|c|}
\hline 年份 & 地区 & 调控目的 & 调控方法 & 生态影响 & 缓解对策 \\
\hline 1991 & $\begin{array}{c}\text { 美国 } \\
\text { 明尼苏达 }\end{array}$ & 航运、渔业等 & $\begin{array}{l}\text { 增加 和 减 少 } \\
\text { 波动幅度 }\end{array}$ & $\begin{array}{l}\text { 生物多样性和覆盖 } \\
\text { 度减少 }\end{array}$ & 增加年际波动方案 \\
\hline 1998 & $\begin{array}{l}\text { 加拿大 } \\
\text { 新斯科舍 }\end{array}$ & 水电 & 高水位 & $\begin{array}{l}\text { 多样性减少,外来种 } \\
\text { 增加,湿生物种减少 }\end{array}$ & $\begin{array}{l}\text { 调整年内波动幅度 } 1 \sim 2 \mathrm{~m} \text {, 年际波 } \\
\text { 动幅度为年内波动幅度的 } 1 / 4 \text { 左右 }\end{array}$ \\
\hline 1999 & $\begin{array}{c}\text { 美国 } \\
\text { 路易斯安那 }\end{array}$ & $\begin{array}{l}\text { 滨海退化湿地 } \\
\text { 的保护 }\end{array}$ & 低水位 & $\begin{array}{l}\text { 不同湿地反应不一, } \\
\text { 多样性上升或下降 }\end{array}$ & $\begin{array}{l}\text { 因地制宜, 对多样性减少的湿地制 } \\
\text { 定新调控方案 }\end{array}$ \\
\hline 2000 & 瑞典北部 & 水电 & 高水位 & $\begin{array}{l}\text { 多样性减少, 外来种 } \\
\text { 增加 }\end{array}$ & 适当减少总蓄水量 \\
\hline 2002 & 荷兰 & 防洪、农业生产 & 低水位 & 生物多样性减少 & 增加波动幅度,偶尔恢复先前水位 \\
\hline 2003 & 荷兰 & $\begin{array}{l}\text { 促进新生湖泊 } \\
\text { 湿地植被发育 }\end{array}$ & 增加波动 & 生物多样性增加 & $\begin{array}{l}\text { 选择夏季(生长季) 降低水位, 促进 } \\
\text { 植被萌发、生长 }\end{array}$ \\
\hline 2005 & 莱茵河流域 & 水利 & 高水位 & $\begin{array}{l}\text { 丰富度减少,易受外 } \\
\text { 来种人侵 }\end{array}$ & 枯水期排水,适当降低水位 \\
\hline
\end{tabular}

与国外相比, 我国的研究起步稍晚, 一些大型控湖、控河工程, 如三峡、南水北调等, 对植被的影响仍处 于严密跟踪调查阶段, 现有部分研究佐证了国外的类似结论, 如 $1 \sim 2$ 年的蓄水过程已经可以形成与之相适 应的湿生植被群落 ${ }^{[74]}$. 另外一些计划中的工程如鄱阳湖控湖等, 仍处于方案论证阶段, 缓解对策也只能是对 预想方案的可能影响而言, 相信这些工程未来的研究结果对于本领域将具有重要的指导意义和参考价值.

\section{4 展望}

水位变化对湿地植被的影响研究已经取得了诸多进展, 但同时也存在很多问题, 值得进一步探讨, 可能 成为今后研究的热点内容,包括:

1) 水位对湿地植被影响的定量机理模型. 目前的定量模型局限于统计结论, 缺乏对机理的深人研究, 而 基于生态水文学的完整的机理模型应当包括湿地的水循环过程、营养物质循环过程和主要优势种的竞争过 程,需结合地理、水文、生态、生物多学科知识、技术共同完成.

2) 自然、人为因素驱动下水位变化对湿地植被影响的差别研究. 在全球气候变化的大环境下, 湿地生态 系统的结构和功能往往会发生不可逆的改变, 如果其所在地同时存在较强人为活动的影响, 则需要对人为 驱动因素和自然驱动因素加以区分. 例如夏季副热带高压北移引起南方降雨减少和控湖、控河工程两者对 长江中下游湿地退化作用的差别研究, 对于客观分析人工控河、控湖等工程带来的生态影响至关重要, 也是 社会关注的热点.

3) 室内模拟水位波动对湿地不同植物种生理、生化指标影响的详细研究. 建立湿地生态水文综合模型 至少需要水位对当地各优势植物种影响的基础数据, 目前的室内模拟只局限于芦苇、香蒲等少数几种常见 湿生植物, 应当充分拓展对湿地植物种的选择空间, 同时可以结合野外调查数据, 以不同植物种生理、生化 指标反应的差异作为优势种之间竞争演替的理论基础.

4) 人工控河、控湖的跟踪观测. 多数的人工控湖、控河工程从实施到运行, 持续时间约为 $10 \sim 20$ 年, 这 对于植被群落演替而言, 时间跨度只能算中等, 而且不少工程在制定了新的缓解措施后, 只运行了数年, 控 湖、控河工程的生态效应仍需要进一步的观测、分析,数十年以上的长期影响尚有待论证.

\section{5 参考文献}

[1 1 吕宪国. 我国湿地研究进展. 地理科学, 1998, 18(4):296-298.

[2] 刘 永, 郭怀诚, 周 丰等. 湖泊水位变动对水生植被的影响机理及其调控方法. 生态学报, 2006, 26 (9): 3117-3126. 
[ 3 ] 徐治国,何 岩, 间百兴等. 营养物及水位变化对湿地植物的影响. 生态学杂志,2006,25(1):87-92.

[4] 严登华,何 岩,邓 伟等.生态水文学研究进展. 地理科学,2001,21(5):467-473.

[ 5 ] Nǒges T, Nǒges P, Laugaste R. Water level as the mediator between climate change and phytoplankton composition in a large shallow temperate lake. International Conference on Limnology of Shallow Lakes, Balatonfured, 2002: 257-263.

[ 6 ] Armstrong W. Aeration in higher plants. Advances in Botanical Research, 1980, 7: 225-232.

[ 7 ] Keddy PA, Reznicek AA. Great lakes vegetation dynamics: The role of fluctuating water levels and buried seeds. Journal of Great Lakes Research, 1986, 12 (1) :25-36.

[ 8 ] Wilson SD, Keddy PA. Plant zonation on a shoreline gradient: physiological response curves of component species. Journal of Ecology, 1985, 73: 851-860.

[ 9 ] Nilsson C, Jansson R. Floristic differences between riparian corridors of regulated and free-flowing boreal rivers. Regulated Rivers: Research and Management, 1995, 11(1) : 55-66.

[10] Hill MN, Keddy PA, Wisheu IC. A hydrological model for predicting the effects of dams on the shoreline vegetation of lakes and reservoirs. Environmental Management, 1998, 22(5) : 723-736.

[11] Wassen MJ, Joost JHJ. In search of a hydrological explanation for vegetation changes along a fen gradient in the Biebrza Upper Basin(Poland). Vegetatio, 1996, 124(2): 191-209.

[12] 田 迅, 卜兆军,杨允菲等. 松嫩平原湿地植被对生境干湿交替的响应. 湿地科学,2004,2(2):122-127.

[13] Rorslett B. An integrated approach to hydropower impact assessment. Hydrobiologia, 1989, 175(1) : 65-82.

[14] Keddy PA, Ellis TH. Seedling recruitment of 11 wetland plant-species along a water level gradient-shared or distinct responses. Canadian Journal of Botany, 1985, 63(10) : 1876-1879.

[15] 张 萌, 倪乐意, 徐 军等. 鄱阳湖草滩湿地植物群落响应水位变化的周年动态特征分析. 环境科学研究, 2013,26 (10) : 1057-1063.

[16] Crawford RMM. Oxygen availability as an ecological limit to plant distribution. Advances in Ecological Research, 1992, 23: $93-185$.

[17] Havens KE. Submerged aquatic vegetation correlations with depth and light attenuating materials in a shallow subtropical lake. Hydrobiologia, 2003, 493(1) : 173-186.

[18] 任 南,严国安. 环境因子对东湖几种沉水植物生理的影响研究. 武汉大学学报: 自然科学版, 1996,42(2): 213-218.

[19] White SD, Ganf GG. The influence of convective flow on rhizome length in Typha domingensis over a water depth gradient. Aquatic Botany, 1998, 62(1) : 57-70.

[20] Sorrell BK, Mendelssohn IA, McKee KL et al. Ecophysiology of wetland plant roots: A modelling comparison of aeration in relation to species distribution. Annals of Botany, 2000, 86(3) : 675-685.

[21] Nielsen SL. A comparison of aerial and submerged photosynthesis in some Danish amphibious plants. Aquatic Botany, $1993,45(1): 27-40$.

[22］谭学界,赵欣胜. 水深梯度下湿地植被空间分布与生态适应.生态学杂志,2006,25(12) : 1460-1464.

[23] Keddy PA, Fraser LH. Four general principles for the management and conservation of wetlands in large lakes: the role of water levels, nutrients, competitive hierarchies and centrifugal organization. Lakes and Reservoirs : Research and Management, $2000,5: 177-185$.

[24] 徐 洋, 刘文治, 刘贵华. 生态位限制和物种库限制对湖滨湿地植物群落分布格局的影响. 植物生态学报, $2009, \mathbf{3 3}$ (3) :546-554.

[25] Middelboe AL, Markager S. Depth limits and minimum light requirements of freshwater macrophytes. Freshwater Biology, $1997,37(3): 553-568$.

[26] Grace JB. Effects of water depth on Typha latifolia and Typha domingensis. American Journal of Botany, 1989, 76(5): $762-768$.

[27] Coops H, Vandenbrink FW, Vandenvelde G. Growth and morphological responses of four helophyte species in an experimental water-depth gradient. Aquatic Botany, 1996, 54(1): 11-24.

[28 ] Vretare V, Weisner SEB, Strand JA et al. Phenotypic plasticity in Phragmites australis as a functional response to water depth. Aquatic Botany, 2001, 69: 127-145.

[29] Blanch SJ, Ganf GG, Walker KF. Growth and resource allocation in response to flooding in the emergent sedge Bolboschoe- 
nus medianus. Aquatic Botany, 1999, 63(2) : 145-160.

[30] Lentz KA, Dunson WA. Water level affects growth of endangered northeastern bulrush, Scirpus ancistrochaetus Schuyler. Aquatic Botany, 1998, 60 (3) : 213-219.

[31] Rea N, Ganf GG. Water depth changes and biomass allocation in two contrasting macrophytes. Australian Journal of Marine and Freshwater Research, 1994, 45(8) : 1459-1468.

[32] Weiher E, Keddy PA. The assembly of experimental wetland plant-communities. Oikos, 1995, 73 (3) : 323-335.

[33] Wu Y, Sklar FH, Ruchey K. Analysis and simulations of fragmentation patterns in the everglades. Ecological Applications, $1997,7(1): 268-276$.

[34] Cui B, He Q, Zhao X. Ecological thresholds of Suaeda salsa to the environmental gradients of water table depth and soil salinity. Acta Ecologica Sinica, 2008, 28(4) : 1408-1418.

[35] 张金屯. 数量生态学. 北京:科学出版社, 2004 .

[36] Wang F, Liang RJ, Yang XL et al. A study of ecological water requirements in Northwest China I: Theoretical analysis. Journal of Natural Resources, 2002, 16(1): 1-8.

[37] Howard RJ, Rafferty PS. Clonal variation in response to salinity and flooding stress in four marsh macrophytes of the northern gulf of Mexico, USA. Environmental and Experimental Botany, 2006, 56(3) : 301-313.

[38 ] Kim JW, Lu Z, Lee H et al. Integrated analysis of PALSAR/Radarsat-1 In SAR and ENVISAT altimeter data for mapping of absolute water level changes in Louisiana wetlands. Remote Sensing of Environment, 2009, 113(11) : $2356-2365$.

[39] Wilcox DA, Xie Y. Predicting wetland plant community responses to proposed water-level-regulation plans for Lake Ontario: GIS-based modeling. Journal of Great Lakes Research, 2007, 33(4) : 751-773.

[40] Hebb AJ, Mortsch LD, Deadman PJ et al. Modeling wetland vegetation community response to water-level change at Long Point, Ontario. Journal of Great Lakes Research,2013, 39(2) : 191-200.

[41] Casanova MT, Brock MA. How do depth, duration and frequency of flooding influence the establishment of wetland plant communities? Plant Ecology, 2000, 147(2) : 237-250.

[42] Nicol JM, Ganf GG, Pelton GA. Seed banks of a southern Australian wetland: the influence of water regime on the final floristic composition. Plant Ecology, 2003, 168(2) : 191-205.

[43] Manel L, Macro C. Effects of water level fluctuations on lakes: an annotated bibliography. Hydrobiologia,2008, 613(1): $171-184$.

[44] Vangeest GJ, Roozen F, Coops H et al. Vegetation abundance in lowland flood plan lakes determined by surface area, age and connectivity. Freshwater Biology, 2003, 48(4) : 758-758.

[45] Riis T, Hawes I. Relationships between water level fluctuations and vegetation diversity in shallow water of New Zealand lakes. Aquatic Botany, 2002, 74(2) : 133-148.

[46] Blanch SJ, Ganf GG, Walker KF. Tolerance of riverine plants to flooding and exposure indicated by water regime. Regulated Rivers : Research and Management, 1999, $15(1)$ : 43-62.

[47] Gacia E, Ballesteros E. The effect of increased water level on Isoetes lacustris L. in Lake Baciver, Spain. Journal of Aquatic Plant Management, 1996, 34 : 57-59.

[48 ] Vandervalk AG, Squires L, Welling CH. Assessing the impacts of an increase in water-level on wetland vegetation. Ecological Applications, 1994, 4(3): 525-534.

[49] 于 丹. 水生植物群落动态与演替的研究. 植物生态学报, 1994,18(4): 372-378.

[50] Laine J, Vasander H, Laiho R. Long-term effects of water level drawdown on the vegetation of drained pine mires in southern Finland. Journal of Applied Ecology, 1995, 33(1) : 785-790.

[51] Coops H, Beklioglu M, Crisman TL. The role of water-level fluctuations in shallow lake ecosystems-workshop conclusions. Hydrobiologia, 2003, $\mathbf{5 0 6}(1): 23-27$.

[52] Nǒges P, Tuvikene L, Nǒges T et al. Primary production, sedimentation and resuspension in large shallow Lake Vortsjarv. Aquatic Sciences, 1999, 61(2): 168-182.

[53] 周万平, 郭晓鸣, 陈伟民等. 南水北调东线一期工程对洪泽湖水生生物及生态环境影响的预测. 湖泊科学, 1994,6 (2) : 131-135.

[54] Pezeshki SR. Wetland plant responses to soil flooding. Quebec City: Symposium on Plant and Organisms in Stressed Wetland Environments, 2000 : 299-312. 
[55] Van OM, Van GN, Maltby E et al. Experimental manipulation of water levels in two French riverine grassland soils. Acta Oecologica-International Journal of Ecology, 2000, 21 (1) : 49-62.

[56] Steinman AD, Ogdahl ME, Weinert M et al. Water level fluctuation and sediment-water nutrient exchange in Great Lakes coastal wetlands. Journal of Great Lakes Research, 2012, 36(4) : 766-775.

[57] Keeland BD, Sharitz RR. The effects of water-level fluctuations on weekly tree growth in a southeastern USA swamp. American Journal of Botany, 1997, 84(1) : 131-139.

[58 ] Lenssen JPM, Menting FBJ, Putten WH et al. Effects of sediment type and water level on biomass production of wetland plant species. Aquatic Botany, 1999, 64(2) : 151-165.

[59] Wantzen KM, Rothhaupt KO, Mörtl M et al. Ecological effects of water-level fluctuations in lakes: an urgent issue. Hydrobiologia, 2008, $\mathbf{6 1 3}(1): 1-4$.

[60] Nilsson C, Jansson R, Zinko U. Long-term responses of river-margin vegetation to water-level regulation. Science, 1997, 276(5313) : 798-800.

[61] Flynn KM, Mendelssohn IA, Wilsey BJ. The effect of water level management on the soils and vegetation of two coastal Louisiana marshes. Wetlands Ecology and Management, 1999, 7(4) : 193-218.

[62] Coops H, Hosper SH. Water-level management as a tool for the restoration of shallow lakes in the Netherlands. Victoria: Conference of the American Society of Limnology and Oceanography (ASLO), 2002 : 193-218.

[63] Toner M, Keddy PA. River hydrology and riparian wetlands: A predictive model for ecological assembly. Ecological Applications $, 1997,7(1): 236-246$.

[64] 胡细英,熊小英. 鄱阳湖水位特征与湿地生态保护. 江西林业科技,2002, (5):1-4.

[65] Cooke GD, Welch EB, Peterson S. Restoration and management of lakes and reservoirs: 3rd. Boca Raton: CRC Press, 2005: 591 .

[66] Wilcox DA, Meeker JE. Disturbance effects on aquatic vegetation in regulated and unregulated lakes in northern minnesota. Canadian Journal of Botany, 1991, 69(7) : 1542-1551.

[67] Jansson R, Nilsson C, Dynesius M et al. Effects of river regulation on river-margin vegetation: a comparison of eight boreal rivers. Ecological Applications, 2000, 10 (1) : 203-224.

[68 ] Coops H, Vulink JT, Vannes EH. Managed water levels and the expansion of emergent vegetation along a lakeshore. Konstanz: Lake Shores 2003 International Conference, $2003, \mathbf{1 0}$ (1) : 203-224.

[69] Hellsten S, Riihimaki J. Effects of lake water level regulation on the dynamics of littoral vegetation in northern Finland. Dublin: 9th International Symposium on Aquatic Weeds, 1996: 85-92.

[70] Litvinov AS, Roshchupko VF. Long-term and seasonal water level fluctuations of the Rybinsk Reservoir and their role in the functioning of its ecosystem. Water Resources, 2007, 34(1) : 27-34.

[71] Wilcox DA. Response of wetland vegetation to the post-1986 decrease in Lake St. Clair water levels: Seed-bank emergence and beginnings of the Phragmites australis invasion. Journal of Great Lakes Research, 2012, 38(2) : 270-277.

[72] Nilsson C, Svedmark M. Basic principles and ecological consequences of changing water regimes: Riparian plant communities. Environmental Management, 2002, 30(4) : 468-480.

[73] Vangeest GJ, Coops H, Roijackers RMM. Succession of aquatic vegetation driven by reduced water-level fluctuations in floodplain lakes. Journal of Applied Ecology, 2005, 42(2):251-260.

[74] 孙 荣, 袁兴中, 丁佳佳. 三峡水库蓄水至 $156 \mathrm{~m}$ 水位后白夹溪消落带植物群落生态学研究. 湿地科学, 2010,8 (1) $: 1-7$. 